\title{
Adaptive-passive control of structure- borne noise of rotating machinery using a pair of shunted inertial actuators
}

Journal of Intelligent Material Systems and Structures

I-16

(C) The Author(s) 2015

Reprints and permissions:

sagepub.co.uk/journalsPermissions.nav DOI: I0.II77//045389XI5600080 jim.sagepub.com (S)AGE

\author{
Guoying Zhao', Neven Alujević', Bruno Depraetere', \\ Gregory Pinte ${ }^{2}$ and Paul Sas'
}

\begin{abstract}
In this article, two piezo-based rotating inertial actuators are considered for the suppression of the structure-borne noise radiated from rotating machinery. Each inertial actuator comprises a piezoelectric stack element shunted with the Antoniou's gyrator circuit. This type of electrical circuit can be used to emulate a variable inductance. By varying the shunt inductance it is possible to realise two tuneable vibration neutralisers to suppress tonal frequency vibrations of a slowly rotating machine. Also, reductions in the noise radiated from the machine housing can be achieved. First, a theoretical study is performed using a simplified lumped parameter model of the system at hand. The simplified model consists of a rotating shaft and two perpendicularly mounted shunted piezo-based rotating inertial actuators. Second, the shunted piezo-based rotating inertial actuators are tested on an experimental test bed comprising a rotating shaft mounted in a frame. The noise is radiated by a plate that is attached to the frame. The experimental results show that a reduction of $\mathrm{II} \mathrm{dB}$ on the disturbance force transmitted from the rotating shaft through the bearing to the housing can be achieved. This also generates a reduction of $9 \mathrm{~dB}$ for the plate vibration and the radiated noise.
\end{abstract}

\section{Keywords}

Piezoelectric element, rotating inertial actuator, shunting, adaptive-passive control, structure-borne noise

\section{Introduction}

Noise radiation from structural housing in rotating machinery is a common problem in many industrial applications, such as gearboxes, compressors, electric motors and so on. In these cases, vibrations of rotating elements, which are transmitted through the bearings to the noise-radiating surfaces such as the machine frame, are often the major noise source. In order to reduce the received noise level, techniques such as sound absorption-based insulation, including encapsulation, can be used to interrupt the airborne sound transmitted from the machine to the environment. These passive sound control techniques would typically be used to deal with noise at higher frequencies. At lower frequencies, where the acoustic wavelength is much larger than the maximum permissible thickness of the insulation layers, active noise control strategies can be considered instead (Elliott et al., 1990, 1992; Kuo and Morgan, 1995; Nelson and Elliott, 1991). These however become more complicated and more expensive, or alternatively less efficient, if the size of the enclosure where the sound is controlled (Elliott et al., 1992) is comparatively large. Lots of error sensors and control actuators are necessary for good control performance, and in fact the total length of the wiring to connect peripheral units to the centralised controller can become a limitation (Elliot et al., 1990). In such situations it could be preferred to directly reduce the vibro-acoustic response of the noise-radiating surfaces. This can be done by applying control forces on the surfaces (Alujević et al., 2011, 2014; Crawley and De Luis, 1987; Dehandschutter, 1997; Fuller et al., 1997; Paulitsch et al., 2004, 2006; Pinte, 2007; Qiu et al.,

\footnotetext{
'Production Engineering, Machine Design and Automation (PMA) Section, KU Leuven, Leuven, Belgium

${ }^{2}$ Flanders Make, Leuven, Belgium
}

\section{Corresponding author:}

Guoying Zhao, Production Engineering, Machine Design and Automation (PMA) Section, KU Leuven, Celestijnenlaan 300, Box 2420, 300I Leuven, Belgium.

Email: nudt.guoying@gmail.com 
2007) or isolating the transmitted force to the surfaces (Chen and Brennan, 2000; Guan et al., 2004, 2005; Li et al., 2005; Pinte et al., 2010; Rebbechi et al., 1999; Stallaert, 2010; Sutton et al., 1997). In case forces are applied directly to the noise radiation surfaces, passive tuned mass dampers (Alujević et al., 2012; Den Hartog, 1985; Hunt, 1979; Inman, 2008), inertial shakers (Alujević et al., 2014; Paulitsch et al., 2004, 2006), reactive actuators (Alujević et al., 2011) or piezoelectric patches (Crawley and De Luis, 1987; Fuller et al., 1997; Qiu et al., 2007) are often employed to produce the control forces. However, this approach may become cumbersome and expensive for large and complex systems which have many radiating surfaces.

On the other hand, in the active vibration isolation approach, it is attempted to block the transmitted vibrations in the structural transfer paths before they reach the noise-radiating surfaces. This may yield a control system that is less complex in case there are concentrated bottlenecks in the vibration transmission paths. With rotating machinery such concentrations typically occur in bearings that support revolving shafts. Several studies based on this approach have been published recently. Rebbechi et al. (1999) proposed to integrate two pairs of magnetostrictive actuators into a double row bearing, which is mounted on the input shaft next to the input pinion, with the aim of actively isolating the vibration transmitted from the shaft to the housing. A reduction of $20-28 \mathrm{~dB}$ can be obtained in the housing vibration at the fundamental gear mesh frequency. Pinte et al. (2010) and Stallaert (2010) adopted a similar approach, but used two piezoelectric actuators instead, which are perpendicularly mounted onto one of the support bearing locations in order to limit the force transmitted from the shaft to the housing. Instead of placing actuators in series with the original bearing, Guan et al. (2005) and Li et al. (2005) proposed to mount control actuators in parallel, directly on the shaft via an additional bearing (active shaft transverse vibration control). In such a case, the system suspension stiffness is not influenced by the introduction of control actuators. Chen and Brennan (2000) presented an inertial actuator control concept, where three magnetostrictive inertial shakers are positioned tangentially at $120^{\circ}$ intervals on the gear body, in order to suppress the gear vibrations at the source. The above-mentioned actuation concepts for the suppression of gearbox housing vibrations are theoretically compared by Guan et al. (2004). In this theoretical study, the actuation effort, control robustness and implementation costs are taken into account as the comparison criteria. The shaft transverse vibration active control approach appeared to be the best compromise regarding the required amplitude of the control force below $500 \mathrm{~Hz}$, while also yielding a reasonable control robustness and a limited implementation cost.
In this study, an axisymmetric piezo-based rotational inertial actuator, which can be installed directly on the rotating shaft as an add-on device, is proposed and studied experimentally. The benefit of this add-on approach is that the machine stiffness is not affected as is the case with, for example, active bearings. Another advantage is the relative ease of implementation in a practical setting as no major structural modification is required. Furthermore, the active element is not in a critical path of the machine such that a possible failure of the piezoelectric element does not necessarily affect the functionality of the machine.

Both active and adaptive-passive methods can be employed to generate appropriate control signals for the piezoelectric actuator in the piezo-based rotating inertial actuator (PBRIA). The active methods can achieve a high control authority, but at the expense of high technical complexity, high costs and lower reliability (Kodejska et al., 2012). Also, the active control methods require a continuous energy supply in order to drive the actuators during the control system operation. A viable alternative approach is to use adaptivepassive methods. These are simpler, cheaper, lighter and easier to implement than the active control means. In such a case, the piezoelectric elements are often used in conjunction with external shunt circuits which do not necessarily consume energy to generate the control force. For example, a change of the electrical boundary conditions of the piezoelectric element generates a change of its mechanical properties such as its effective stiffness and effective damping. Date et al. (2000) have shown that the effective elastic modulus and the damping of poled polyvinylidene fluoride samples can be modified by connecting a negative capacitance and a resistance, respectively. Davis and Lesieutre (2000) developed a tuneable solid-state piezoelectric vibration absorber, where a piezoelectric transducer is incorporated in the suspension of the passive absorber. The resonance frequency of the developed device is thus partly determined by the stiffness of the piezoelectric element. By altering the positive switching shunt capacitance, the stiffness of the piezoelectric element is altered, leading the absorber to have a $\pm 3.7 \%$ tuneable frequency band. Apart from capacitance shunt circuits, shunt circuits with inductors and resistors connected in series have also been investigated (Hagood and Von Flotow, 1991; Hollkamp, 1994; Hollkamp and Starchville, 1994; Lesieutre, 1994; Niederberger, 2005; Park and Inman, 2003). This type of shunt circuit generates an electrical resonance with the already present piezoelectric capacitance. The operation of such a device is often compared to a mechanical tuned mass damper, where the shunt inductor and resistor behave as an additional mass and damper, respectively. If the aim is to attenuate harmonic vibrations, a vibration neutraliser, which can introduce an anti-resonance at the frequency of the tonal disturbance to the driving 
point receptance of the host structure, is more appropriate than a tuned mass damper. At resonances, the responses normally decrease when increasing the neutraliser damping, but at the anti-resonance the response increases with an increase in damping. For harmonic disturbances, it is thus preferred to keep the damping in the neutraliser suspension light. This consequently implies that the resistance in the shunt circuit should be reduced in order to realise maximum vibration suppression in a narrow frequency band, meaning that only a capacitance or an inductance should be attached to the PBRIA. For the capacitance shunt, the effective stiffness of the piezoelectric actuator in the PBRIA can be altered by the shunt capacitance such that the natural frequency of the PBRIA can be tuned to the frequency of concern. In this way, the capacitance-shunted PBRIA behaves exactly the same as a vibration neutraliser. However, a negative capacitance might be needed depending on ratio of the natural frequency of PBRIA and the disturbance frequency, so that care must be taken with the stability of the system and a limited robustness for the resultant system can be expected (Devos and Pinte, 2009). This can be avoided using the inductive shunt circuits, where a positive inductance is used and thus no stability problems may occur, assuming a perfect inductance circuit (Neubauer et al., 2006). Because of this advantage, an inductive shunt is used in this study. It is connected to the piezoelectric actuator in the PBRIA in order to generate a neutralising effect at a single frequency. By relying on a variable inductance, the neutraliser frequency can be tuned to the frequency of the tonal disturbance.

One of the aims of this article is to investigate whether or not it is feasible to suppress structure-borne noise/vibration by attaching shunted PBRIAs directly onto a slowly rotating shaft. In section 'Design of the piezo-based rotating inertial actuator', the design of the PBRIA is briefly covered. In the following section, a bidirectional lumped parameter model of a simple system is derived, on which the theoretical performance of the control strategy is predicted. In section 'Experimental validation', the experimental test bed used to evaluate the performance of the developed PBRIA is first described, and then the experimental results of the implemented control strategy are presented and analysed. Section 'Conclusion' summarises the main conclusions of the article.

\section{Design of the piezo-based rotating inertial actuator}

Figure 1 shows the developed prototype of the piezobased rotating inertial actuator. The idea behind the design of the PBRIA is to use a piezoelectric actuator to introduce a force between a rotating element (e.g. the shaft) and a ring-shaped mass rotating together

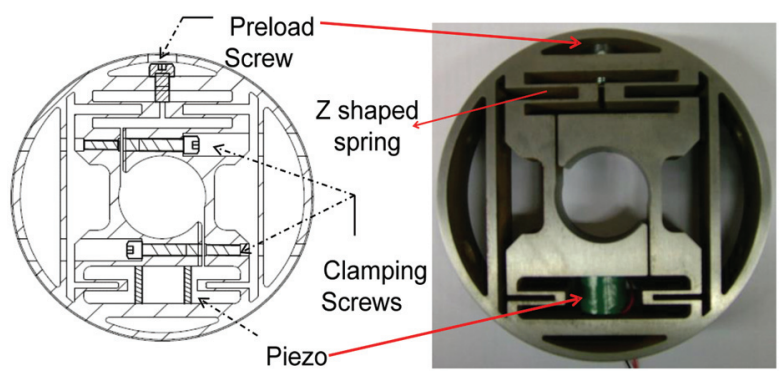

Figure I. Developed piezo-based rotating inertial actuator.

with the shaft. By accelerating the ring-shaped mass along the actuation direction of the piezoelectric actuator, compensating forces can be generated on the shaft. The Piezomechanik HPSt 150/20 piezoelectric stack actuator is used. Although the developed PBRIA has a sufficient force capacity to compensate the disturbances on the test bed in the frequency range of interest (200 $1000 \mathrm{~Hz}$ ), it is acknowledged that in industrial applications, where excitation forces are larger, longer piezoelectric actuators or heavier proof mass should be used to generate the required compensating force.

In order to eliminate the need for an additional fixture mechanism, the inner collar as shown in Figure 1 is split. Moreover, the splits are offset in order to prevent stress concentration on the piezoelectric actuator. Note that the maximum applicable negative voltage for the piezoelectric stack actuator is much smaller than the maximum applicable positive voltage, so that the piezoelectric stack actuator cannot withstand a high level of tension force. In practice, this issue can be solved by applying a preload force to the piezoelectric actuator. In this design, the piezoelectric actuator is preloaded by the flexures (two Z-shaped springs) on the other side, such that it is capable of applying bi-directional (push/ pull) forces. A cap screw is then used to tighten the Zshaped springs and a pre-stress of approximately $250 \mathrm{~N}$ is applied to the piezoelectric element.

The proof mass (outer ring) of the PBRIA is suspended by four Z-shaped springs. These springs are designed to realise a low translational stiffness in one radial direction (vertical direction in Figure 1), while guaranteeing a high stiffness in the tangential direction (horizontal direction in Figure 1) as well as high torsional stiffness. A high torsional stiffness is designed in order to avoid excessive bending stress for the piezoelectric actuator in the low frequency range.

From the piezoelectric shunting control point of view, the design of the PBRIA should maximise the generalised electromechanical coupling coefficient at the considered structural resonance frequency. This parameter measures the percentage of total system modal strain energy actually converted into electrical energy by the piezoelectric element, which is principally determined by the properties of the device such as the 
fundamental resonance frequency, the proof mass and the piezoelectric coupling coefficient. The proposed PBRIA utilises a piezoelectric stack actuator which yields a high piezoelectric coupling coefficient and also associates a high fundamental resonance frequency around $1300 \mathrm{~Hz}$. Since the generalised electromechanical coupling coefficient serves the same function as the mass ratio in the tuned mass damper system (Hagood and Von Flotow, 1991), a large value of this parameter is beneficial. For the proposed PBRIA, the value of the generalised electromechanical coupling coefficient is inversely proportional to the ratio between the resonance frequency of the PBRIA and that of the primary structural (Zhao et al., 2014). A high resonance frequency of the PBRIA implies a large resonance frequency ratio, which reduces the generalised electromechanical coupling coefficient and thus reduces its effectiveness in the low frequency range. Alternatively, a $d_{31}$ piezoelectric effect could be used in the future in conjunction with bending beams, which is referred to as bimorph actuation technology, to accelerate the outer ring mass (see, for example, Alujevic et al., 2012). As such, it is possible to realise a PBRIA with a low fundamental resonance frequency. However, one should bear in mind that the piezoelectric coupling coefficient for the $d_{31}$ mode is reported to be two times smaller than for the $d_{33}$ mode (Preumont, 2002). Since both design concepts have advantages and disadvantages, the actuating mechanism of choice for a PBRIA in terms of using piezoelectric shunting control will be problem specific.

\section{Modelling and working principle}

In this section, a model of a simplified system, which consists of a rotating shaft and two perpendicularly mounted resonantly shunted PBRIAs, is presented in order to predict the theoretically achievable performance. The bi-directional lumped parameter model of the considered system is shown in Figure 2. The



Figure 2. Bi-dimensional lumped parameter model of the considered system. rotating shaft, represented by the mass $m_{1}$, is connected to the ground by two spring-dashpot pairs. As a consequence, the shaft is assumed to only vibrate in the horizontal direction $(x)$ and vertical direction $(y)$. The PBRIA, in which the piezoelectric actuator orients $\theta$ degrees from the $x$ axis, is defined through one proof mass $m_{2}$, one spring-dashpot pairs $\left(k_{2}, c_{2}\right)$ and one shunted piezoelectric actuator. The proof mass is assumed to only vibrate radially. $k_{2}$ physically represents the stiffness of the leaf springs. The piezoelectric actuator is placed parallel to the leaf spring-dashpot pair. The second PBRIA is modelled identically, but placed perpendicularly with respect to the first PBRIA as shown in Figure 2. A small amount of damping is introduced to have a damping ratio of $1 \%$. A disturbance $f_{d}$ is assumed to directly act on the shaft along the $y$ axis and keep in this orientation while the shaft spins. Furthermore, it is assumed that the rotation speed is slow and much smaller than the frequency of disturbance, thereby centrifugal and Coriolis forces are not considered. These assumptions are valid, for example, in wind turbine applications where gearboxes are typically used to connect a low-speed shaft spinning at about 30-60 r/min to a high-speed shaft rotating at about $1000-1800 \mathrm{r} / \mathrm{min}$. In such applications, due to the variation of the teeth stiffness and the transmission error, certain dynamic disturbances are generated, the orientation of which keeps constant (along the line of gear mesh actuation) during the rotation of the shaft. The resulting vibration/acoustic response is dominated by narrow-band peaks at the gear mesh frequency (given by the product of the number of the teeth and shaft speed) and its harmonics. Thus, it is expected that the disturbance frequency is much larger than the rotation speed. Also, a perfect mechanical decoupling is assumed so that disturbing forces of the actuator in one direction are sensed only by the collocated sensor and not by the sensor in the perpendicular direction. In practice, however, some coupling is inevitable. It is nevertheless useful to have the idealised model described here as a benchmark case.

The governing equations of the considered system under the stated assumptions can be written as

$$
\begin{aligned}
{[M]_{\omega_{0} t}\{\ddot{u}\} } & +[C]_{\omega_{0} t}\{\dot{u}\}+[K]_{\omega_{0} t}\{u\} \\
& +\left\{F_{p}\left(\dot{u}, u, \omega_{0} t\right)\right\}=\left\{F_{d}(t)\right\}
\end{aligned}
$$

with $\omega_{0}$ is the rotation speed, $[M]_{\omega_{0} t},[K]_{\omega_{0} t}$ and $[C]_{\omega_{0} t}$ are the angular position-dependent (time variant) mass, stiffness and damping matrices, $\left\{F_{p}\left(\dot{u}, u, \omega_{0} t\right)\right\}$ is the force vector generated by the piezoelectric actuators, $\left\{F_{d}(t)\right\}$ is the disturbance force and $\{u\}$ is the vector of the spatial displacements.

In order to further simplify the analysis, equation (1) is rewritten in terms of an equivalent quasi-static condition 
$[M]_{\theta}\{\ddot{u}\}+[C]_{\theta}\{\dot{u}\}+[K]_{\theta}\{u\}+\left\{F_{p}(\dot{u}, u, \theta)\right\}=\left\{F_{d}(t)\right\}$

where $\theta$ is used to substitute $\omega_{0} t$. Thus, a linear differential equation with time-invariant coefficients is obtained.

The linear differential equation given by equation (2) approximates the motion of the system by neglecting the harmonic frequency components caused by the rotation of the shaft.

Equation (2) is then rewritten in the Laplace domain yielding

$$
[M]_{\theta}\{u\} s^{2}+[C]_{\theta}\{u\} s+[K]_{\theta}\{u\}+\left\{F_{p}(s)\right\}_{\theta}=\left\{F_{d}(s)\right\}
$$

Expressions for the matrices $[M]_{\theta},[C]_{\theta}$ and $[K]_{\theta}$ and vectors $\{u\},\left\{F_{p}(s)\right\}_{\theta}$ and $\left\{F_{d}(s)\right\}$ are given in Appendix 1.

With equation (3), the values of the shunt inductances to reduce the shaft vibrations excited at a single short-circuit stiffness of the piezoelectric actuator and the ratio of the electrical impedance of the piezoelectric capacitance to the electrical impedance of the external shunt circuit, respectively.

The $\alpha(s)$ term in equation (5) allows the effective stiffness of the piezoelectric element, $k_{p}^{*}$, to be tuned by changing the electrical impedance of the external shunt circuit. In this article, the inductance-resistance type of shunting is used to examine the effect of the proposed control approach. The ratio $\alpha(s)$ is thus defined as

$$
\alpha(s)=\frac{1}{s C_{s 1}\left(s L_{1}+R_{1}\right)}
$$

where $L_{1}$ and $R_{1}$ denote the inductance and the inherent resistance of the shunt, $C_{S 1}$ is the capacitance of the piezoelectric stack with no external load.

Using the expression in equation (4) to represent the reaction force of the shunt piezoelectric actuator, the driving point receptance of the considered 3-degree-offreedom system is derived

$$
\frac{y_{1}}{F_{d}}=\frac{m_{2} s^{2}+c_{2} s+k_{2}+k_{p 1}^{*}}{\left(\left(m_{1}+m_{3}\right) s^{2}+c_{1} s+k_{1}\right)\left(m_{2} s^{2}+c_{2} s+k_{2}+k_{p 1}^{*}\right)+\left(c_{2} s+k_{2}+k_{p 1}^{*}\right) m_{2} s^{2}}
$$

frequency can be determined. These shunt values are obtained by minimising the numerator of the driving point receptance of the shaft (transfer function between $y_{1}$ and $F_{d}$ ). Assuming that the cross-coupling of the PBRIA is negligible, the order of the governing matrix as given in equation (3) is $2 \times 2$ when one of the piezoelectric actuators in the PBRIAs is parallel to the disturbance. By setting the real part of the numerator of the driving point receptance of the resulting system to zero, it is possible to obtain an explicit expression for the optimal shunt inductance. Afterwards, the optimal value of the other shunt inductance can be calculated in the same way by rotating the shaft by $90^{\circ}$. The obtained two values of the inductance shunts are kept fixed during the rotation, which is the control strategy used in the article.

For one shunted piezoelectric actuator, the reaction force produced by the piezoelectric element is expressed by (Date et al., 2000)

$$
f_{p}=k_{p}^{*} \Delta l
$$

where $\Delta l$ is the effective stroke and $k_{p}^{*}$ is the complex stiffness of the piezoelectric actuator which is given by

$$
k_{p}^{*}=k_{p}\left[\frac{1+\alpha(s)}{1+\alpha(s)-k^{2}}\right]
$$

This complex stiffness given by equation (5) is in function of the following parameters $k, k_{p}$ and $\alpha(s)$, which are the piezoelectric coupling coefficient, the
Substituting equations (5) and (6) into equation (7) and solving the real part of its numerator for $L_{1}$ yields

$$
L_{1}^{\mathrm{opt}}=\frac{\left(\left(1-k^{2}\right) c_{2} R_{1} C_{s 1}+m_{2}\right) \omega^{2}-k_{m 1}-k_{2}}{\left(\left(1-k^{2}\right) m_{2} \omega^{2}-k_{2}-k_{m 1}+k_{2} k^{2}\right) C_{s 1} \omega^{2}}
$$

Following the same procedure, the optimal inductance for the other PBRIA is derived as, assuming the system has rotated such that the PBRIA with mass $m_{3}$ is oriented along the $y$-direction

$$
L_{2}^{\mathrm{opt}}=\frac{\left(\left(1-k^{2}\right) c_{3} R_{2} C_{s 2}+m_{3}\right) \omega^{2}-k_{m 2}-k_{3}}{\left(\left(1-k^{2}\right) m_{3} \omega^{2}-k_{3}-k_{m 2}+k_{3} k^{2}\right) C_{s 2} \omega^{2}}
$$

The control effect of the resonantly shunted PBRIA is examined in terms of the vibration reductions at the resonance frequency of the base structure. This is done by calculating the difference in the amplitude of the driving point receptance of the system described in equation (3) in two cases: (1) the PBRIAs are open circuit connected and (2) the PBRIAs are shunted with the inductances calculated by equations (8) and (9), respectively. The governing equation (3) is solved numerically using the parameters given in Table 1 .

The simulation has been carried out in three conditions: (1) only one resonantly shunted PBRIA is activated, (2) two identical resonantly shunted PBRIAs are activated, (3) two resonantly shunted PBRIAs with $10 \%$ variation in the proof mass and the leaf stiffness (the proof mass $m_{3}$ is 1.1 times larger than $m_{2}$, the leaf stiffness $k_{3}$ is $10 \%$ less than $k_{2}$ ) are activated. For the 
Table I. Definition of the system shown in Figure 2.

\begin{tabular}{ll}
\hline Parameter & Value \\
\hline Mass, $m_{1}$ & $2.9 \mathrm{~kg}$ \\
Proof mass of the PBRIAs, $m_{2}=m_{3}$ & $0.2 \mathrm{~kg}$ \\
Base stiffness, $k_{1}=k_{4}$ & $3.7 \times 10^{7} \mathrm{~N} / \mathrm{m}$ \\
Leaf stiffness, $k_{2}=k_{3}$ & $2.4 \times 10^{6} \mathrm{~N} / \mathrm{m}$ \\
Piezoelectric coupling coefficient, $k$ & $0.28(-)$ \\
Piezoelectric actuator stiffness, $k_{p 1}=k_{p 2}$ & $1.1 \times 10^{7} \mathrm{~N} / \mathrm{m}$ \\
Piezoelectric capacitance, $C_{s 1}=C_{s 2}$ & $2.6 \times 10^{-6} \mathrm{~F}$ \\
Resistance, $R_{1}=R_{2}$ & $3 \Omega$ \\
Shunt inductance, $L_{1}^{\text {opt }}$ and $L_{2}^{\text {opt }}$ & $C$ Calculated by equations $(8)$ and $(9)$ \\
Damping, $c_{1}=c_{4}, c_{2}=c_{3}$ & $c_{1}=207.2 \mathrm{Ns} / \mathrm{m}, c_{2}=67.1 \mathrm{Ns} / \mathrm{m}$
\end{tabular}

PBRIA: piezo-based rotating inertial actuator.

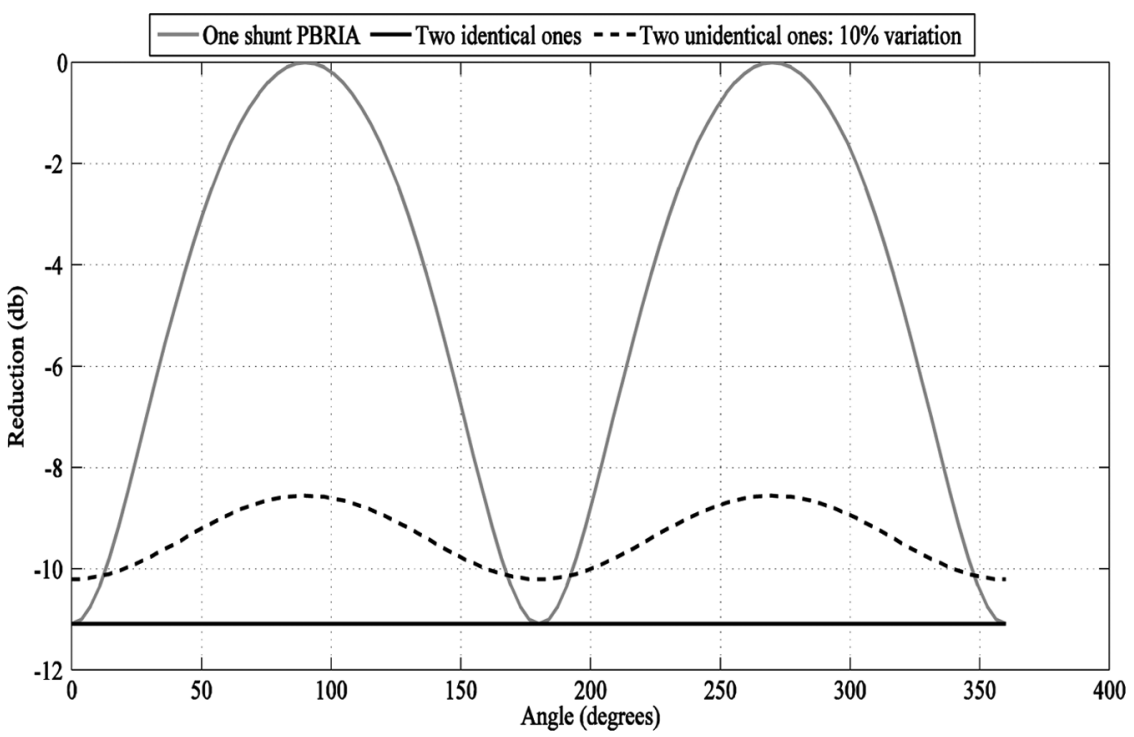

Figure 3. Comparison of the achievable reduction in the case of using one resonantly shunted PBRIA, two identical PBRIAs and two PBRIAs with $10 \%$ variation (the proof mass $m_{3}$ is 1.1 times larger than $m_{2}$, the leaf stiffness $k_{3}$ is $10 \%$ less than $k_{2}$ ).

three cases, the achievable reductions are plotted with respect to the angular position in Figure 3. It is seen that the achievable reductions for the first case behave as a periodic function in terms of the angular position with a period of $\pi$. The maximum reduction is obtained when the piezoelectric actuation direction of this PBRIA is parallel to the disturbance and no reduction is achieved when the actuation direction is perpendicular to the disturbance. For the second case, a constant reduction of $11 \mathrm{~dB}$, which is the same as the maximum reduction obtained for the first case, is achieved. For the third case, the achieved reduction is not constant anymore and shows an oscillation with a period of $\pi$. Note that the maximum achievable reduction is different for the last two cases, which is attributed to the shift of the base structure resonance frequency resulting from the altered PBRIA, while the reduction for both cases is still calculated at the resonance frequency for the second case. As a result, the max achievable reduction for the third case is no longer at this frequency and thereby it is slightly smaller than that for the second case.

In practice, the variation of the bearing stiffness also impacts the control effect of the resonantly shunted PBRIAs, which is, however, assumed to be fixed in the simulation. The simulation results will be still valid in the case the variation level of the bearing stiffness is comparably small to the suppressing band introduced by the resonant shunt.

\section{Experimental validation}

In order to demonstrate the feasibility of the proposed active structural acoustic control approach, a representative setup for rotating machinery is employed, which is shown in Figure 4. The test bed consists of a shaft which is mounted in a frame by a cylindrical bearing at one side and a double angular contact ball bearing at 

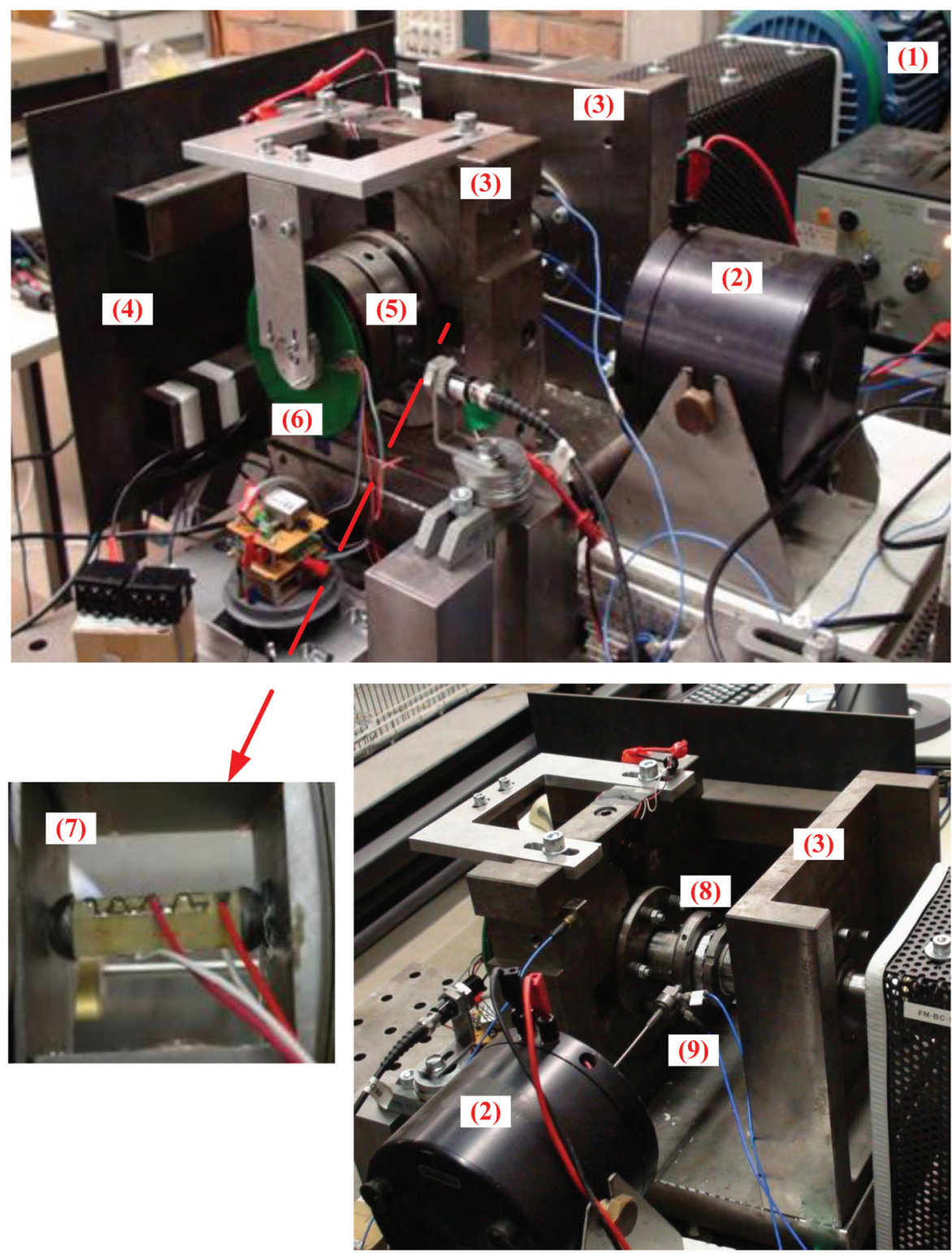

Figure 4. Experimental setup for evaluating the performance of the developed inertia shaker: (I) motor, (2) disturbance shaker, (3) frame, (4) noise radiating plate, (5) PBRIAs, (6) slip ring, (7) force sensor, (8) roller bearing and (9) impedance head.

the other side. This latter bearing is mounted in a ringshaped module in which two piezoelectric sensors are installed to measure the transmitted forces between the shaft and the frame. Close to this bearing, two PBRIAs are perpendicularly mounted on the shaft such that control forces can be generated in all directions while the shaft rotates. The piezoelectric actuators of the two PBRIAs are connected to two synthetic inductance circuits, each of them is realised with a single TCA0372 power operational amplifier as an impedance gyrator, based on the layout in Niederberger (2005). Since these circuits are currently not rotating, a slip ring is equipped and mounted on the shaft close to the PBRIAs to connect them to these circuits.

An electric motor is connected to the shaft to drive the shaft. A metal plate with dimensions
$40 \mathrm{~mm} \times 30 \mathrm{~mm} \times 2 \mathrm{~mm}$ (length $\times$ height $\times$ width) is connected to the frame using two square beam profiles and acts as a noise radiator. The dimensions of the different parts are chosen such that the test bed has a representative dynamic and acoustic behaviour for industrial rotating machinery such as gearboxes (Stallaert, 2010).

In rotating machinery, the disturbance force can be, for example, caused by unbalance, misalignment of the shaft or a mechanical process such as meshing of gears in gearboxes and forces generated by a rotational pump or compressor. In the employed setup, the disturbance force is artificially generated by an electro-dynamic shaker that is connected to the shaft via roller bearing. With the roller bearing, it is possible to introduce arbitrary disturbance forces during the rotation of the 




Figure 5. A cross-sectional view of the experimental setup with all measurement.

shaft. The disturbance force is space fixed and thus may be considered representative of a gear mesh force. Between the roller bearing and the electro-dynamic shaker, an impedance head (PCB 288D01) is placed, which measures the force and acceleration at the force input location.

A number of sensors are installed on and around the test bed in order to evaluate the performance of the resonantly shunted PBRIA. The layout of the sensor configuration is shown in Figure 5, where eight accelerometers are used to measure the structural vibrations, one microphone is used to register the noise level in front of the plate at a distance of approximately $30 \mathrm{~cm}$ and one force gauge is placed between the bearing close to the PBRIA and the frame is used to record the transmitted force. For the accelerometers, four of them are placed on the frame, three are on the shaft and one is mounted in the middle of the plate. The signals from the force gauge, the accelerometers and the microphone are recorded by a dSPACE 1006 system at a sampling frequency of $2.5 \mathrm{kHz}$.

The experiments have been carried out in two conditions: (1) when the shaft is not rotating and (2) when the shaft is rotating. In the first case, only one PBRIA (PBRIA 1, closed to the slip ring) is mounted on the shaft, where the orientation of the piezoelectric actuator is in line with the disturbance shaker. In the second case, two PBRIAs are mounted and slowly rotating together with the shaft and then tests are performed with only a single or both PBRIAs being activated. Before examining the performance of the inductanceshunted PBRIAs, the influence of mounting passive PBRIAs (the electrodes of the piezoelectric elements are open circuited) to the dynamics of the system is first assessed. This is done by comparing the resultant driving point accelerance (frequency response function between the excitation force input to the setup and the acceleration measured at the disturbance input location) of the system when mounting (1) no PBRIAs, (2) one PBRIA and (3) PBRIAs. The comparison is shown in Figure 6. As can be seen, the resonance frequencies associated with the rigid body modes of the shaft (bouncing/even and rocking modes) decrease as PBRIAs are mounted onto the shaft. This is because the PBRIA acts as a rigid mass below its first deformation resonance $(1300 \mathrm{~Hz})$ and this mass is not negligible with respect to the mass of the shaft. However, the anti-resonance of the shaft is almost not affected, which is due to the fact that this frequency is determined by the bearing stiffness and the mass of the frame which are not changed by mounting PBRIAs. Between the shaft anti-resonance and resonance frequencies, some plate modes can be identified, which are characterised by a high modal density and are only slightly affected by the installation of PBRIAs. Details on the modal analysis of the experimental test bed can be found in Zhao (2015).

In this research, the reduction performance is evaluated at the resonance frequencies of the system after mounting the PBRIA/PBRIAs. More importantly, the achieved reductions are obtained when the piezoelectric elements in the PBRIAs are either mistuned or optimally tuned, thereby the passive effect of the PBRIA does not contribute to the reductions reported in the article.

\section{Non-rotating tests}

The potential of the control approach is demonstrated in the case that a sinusoidal disturbance force is applied 


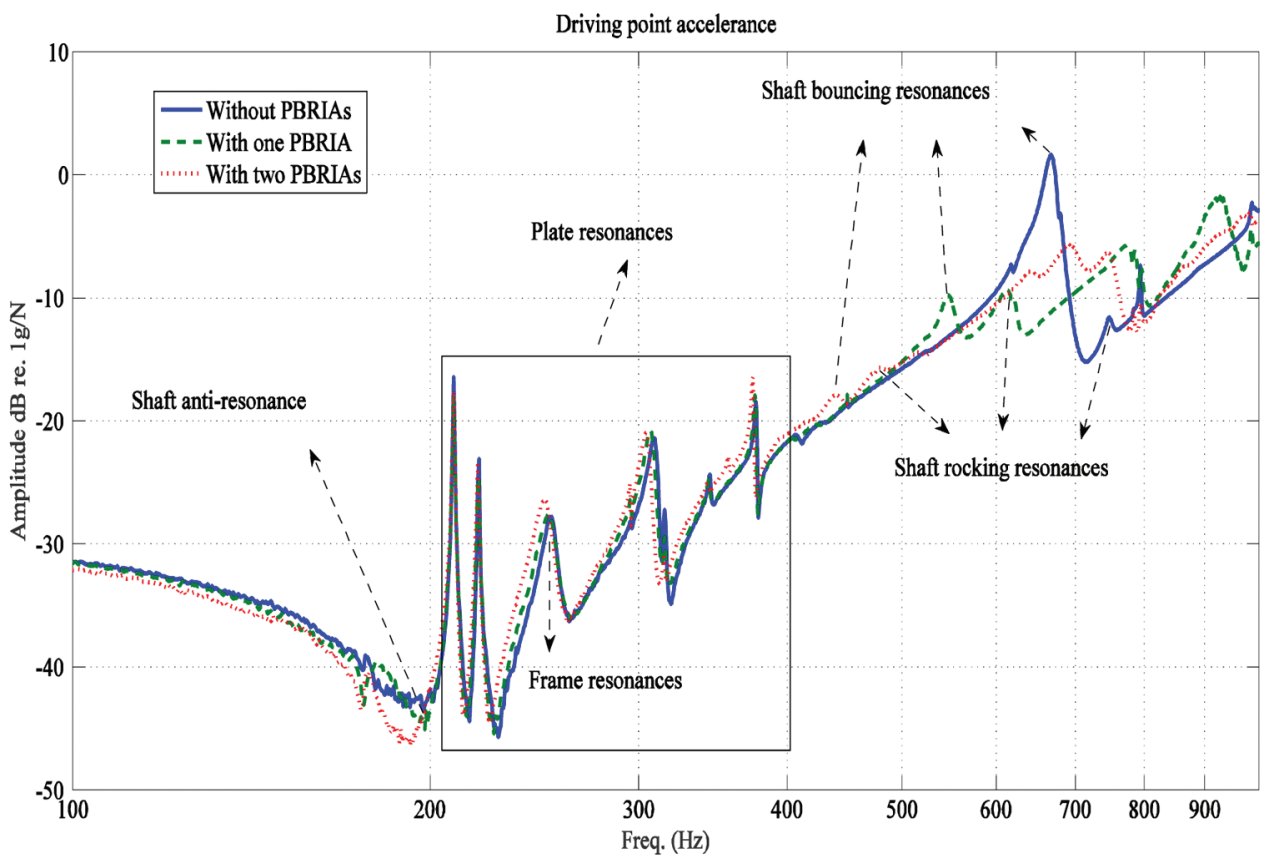

Figure 6. Driving point accelerance measured by the impedance head at the disturbance force input location; the input is the force measured at the input location and the output is the acceleration measured in the same point.

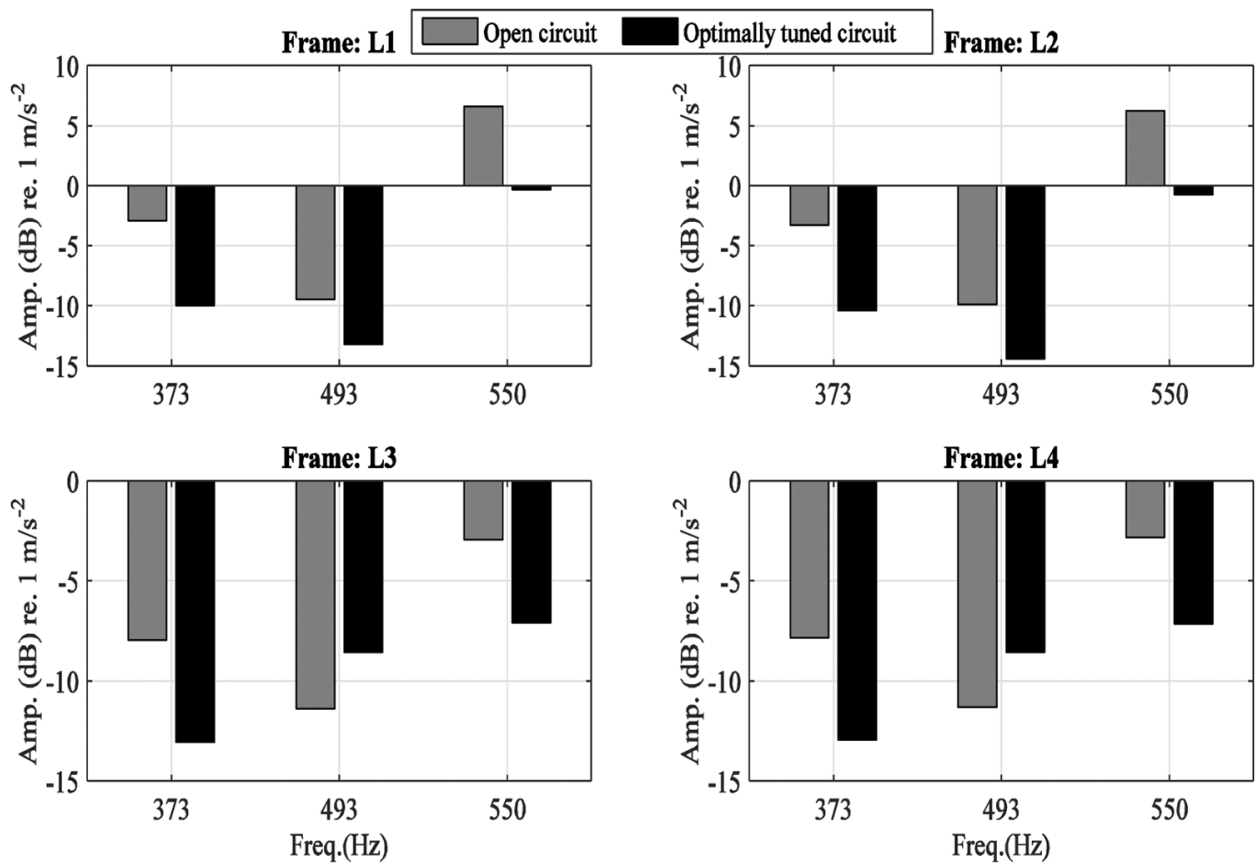

Figure 7. Comparison of the frame accelerations.

by the electro-dynamic shaker at 373, 493 and $550 \mathrm{~Hz}$, respectively. These three frequencies correspond to the plate resonance at which the noise is radiated the most, a random frequency (not structural resonance or antiresonance) and the resonance frequency of the rigid body bouncing mode (even mode) of the shaft, respectively. The appropriate shunt inductances for the
PBRIA 1 to suppress the vibration response at these frequencies are experimentally determined and resulted in the following values: $0.0772,0.0448$ and $0.0364 \mathrm{~Hz}$, respectively. Figures 7 and 8 plot the measured shaft and housing vibration levels obtained with disturbances at the frequencies described above. It can be seen that the dynamic responses are reduced at all the measured 



Figure 8. Comparison of the shaft accelerations.

points at 373 and $550 \mathrm{~Hz}$ when the shunt circuit is optimally tuned. On the contrary, at $493 \mathrm{~Hz}$, the vibration levels, measured at some locations (shaft: L3, frame: L3 and frame: L4), are amplified, while the responses at other measured points are still being attenuated but less than at the other two frequencies. Here, it is of interest to note that the amplification happens at the locations measured far away from the location of the PBRIA, while reductions are achieved close to the PBRIA. It can also be seen that the reductions decrease as the distance between the measured point and the location of the PBRIA increases. This phenomenon might be caused by the fact that the frequency of the rigid body rocking mode of the shaft lies in the same range as the resonance frequency of the rigid body bouncing mode of the shaft, causing the bouncing and rocking motions to be strongly coupled. At the considered non-resonant frequency, the shaft motion is determined by a superposition of the rocking mode and the bouncing mode of the shaft (Zhao, 2015). With the control actuation introduced by the PBRIA, the contribution of the rocking mode might be more pronounced than that of the bouncing mode to the response at the locations that are far away from the location of the PBRIA. As a consequence, certain amplification is observed at these locations. This can, however, be solved by choosing an appropriate location of the PBRIA, which in principle should be as close as possible to the disturbance. An alternative is to mount another PBRIA on the other side of the shaft.

The control effect on the transmitted force, plate vibration and noise radiation levels is shown in Figure 9. It is clear that a reduction of the transmitted force leads to a reduction in the plate vibrations. Depending on the sound radiation efficiency of the plate at each frequency, this leads to a different reduction in the audible noise. For example, a reduction of $9 \mathrm{~dB}$ of the radiation noise is obtained at $373 \mathrm{~Hz}$ (plate resonance frequency), but there are almost no noise reductions observed at the other frequencies.

Based on Figures 7 to 9, one can conclude that the resonantly shunted PBRIA can be tuned to suppress shaft vibrations due to a tonal disturbance by adjusting the value of the electric shunt impedance. This causes the disturbance force transmitted through the bearings to be attenuated, which then leads to a reduction of the housing vibration and ultimately the radiated noise. Higher reductions are, however, obtained for the vibrations of the shaft than for those of the housing. It has also been shown that the location the PBRIA is placed should be considered carefully, and it should ideally be placed as close as possible to the disturbance.

\section{Rotating tests}

In this subsection, experiments with the proposed resonantly shunted PBRIA are performed when the shaft rotates at $60 \mathrm{r} / \mathrm{min}$. An encoder with a resolution of 1024 pulses per revolution is used to measure the rotation of the shaft, the signals from which are then processed by the dSPACE control broad 3001 to calculate the angular position of the shaft. The control effect of the resonantly shunted PBRIA is examined in the case of a sinusoidal disturbance force excitation at $372 \mathrm{~Hz}$. The optimal shunt inductances for the two PBRIAs are 



Figure 9. Comparison of transmitted force, plate vibration and noise radiation.
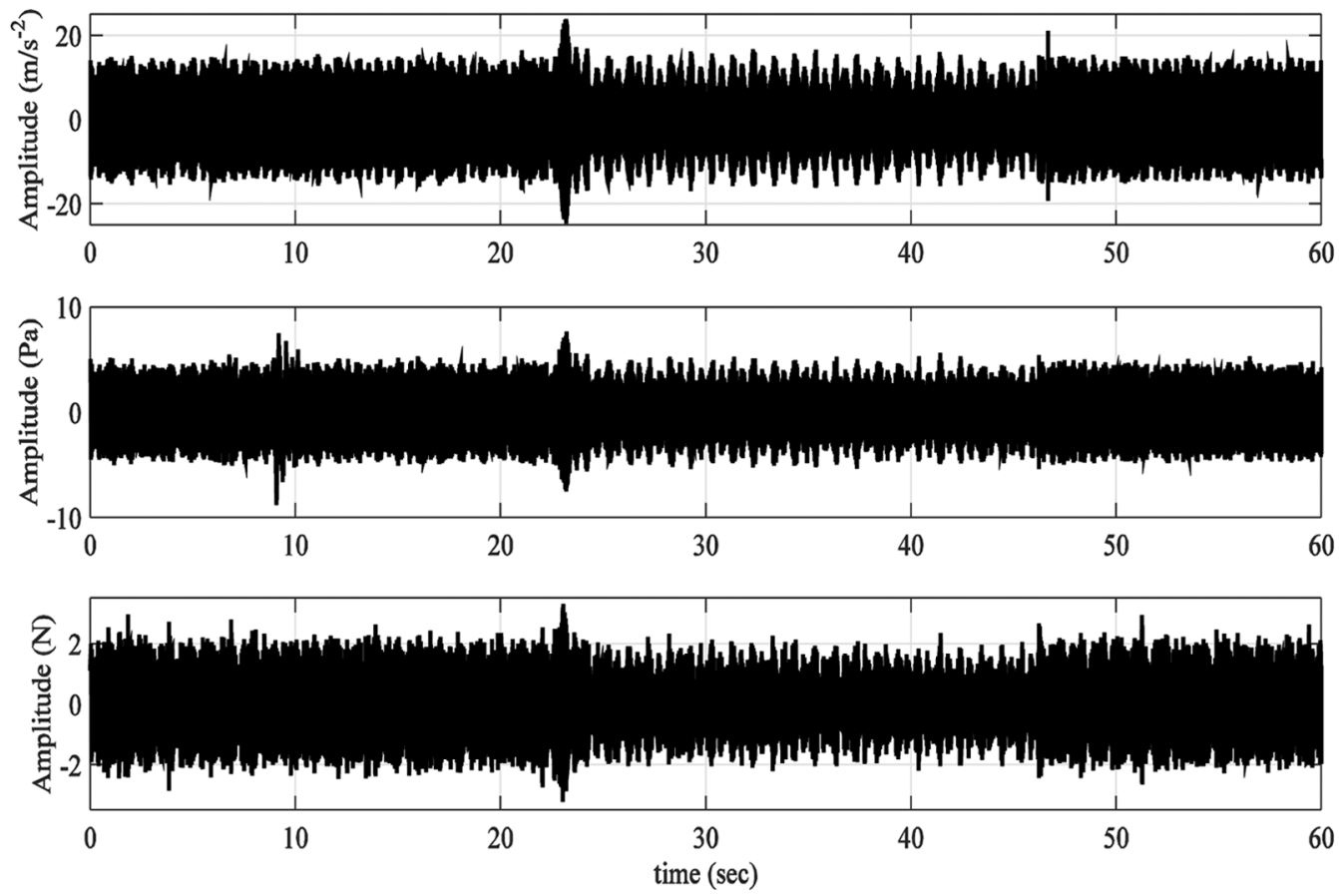

Figure 10. Effect of tuning the inductance connected to the PBRIA on, from top to bottom, the plate acceleration, the transmitted force and the radiated noise in the time domain.

experimentally determined and resulted in the following values: 0.0779 and $0.069 \mathrm{H}$, respectively.

In the first set of experiments, only one resonantly shunted PBRIA is activated and the other one is open circuited. The initial angular shaft position (defined to be $0^{\circ}$ ) corresponds to the dominant actuation direction of the activated PBRIA being perpendicular to that of the disturbance excitation. Figure 10 plots the plate vibration, transmitted force through the bearing close to the PBRIA and the radiated noise, first with the 


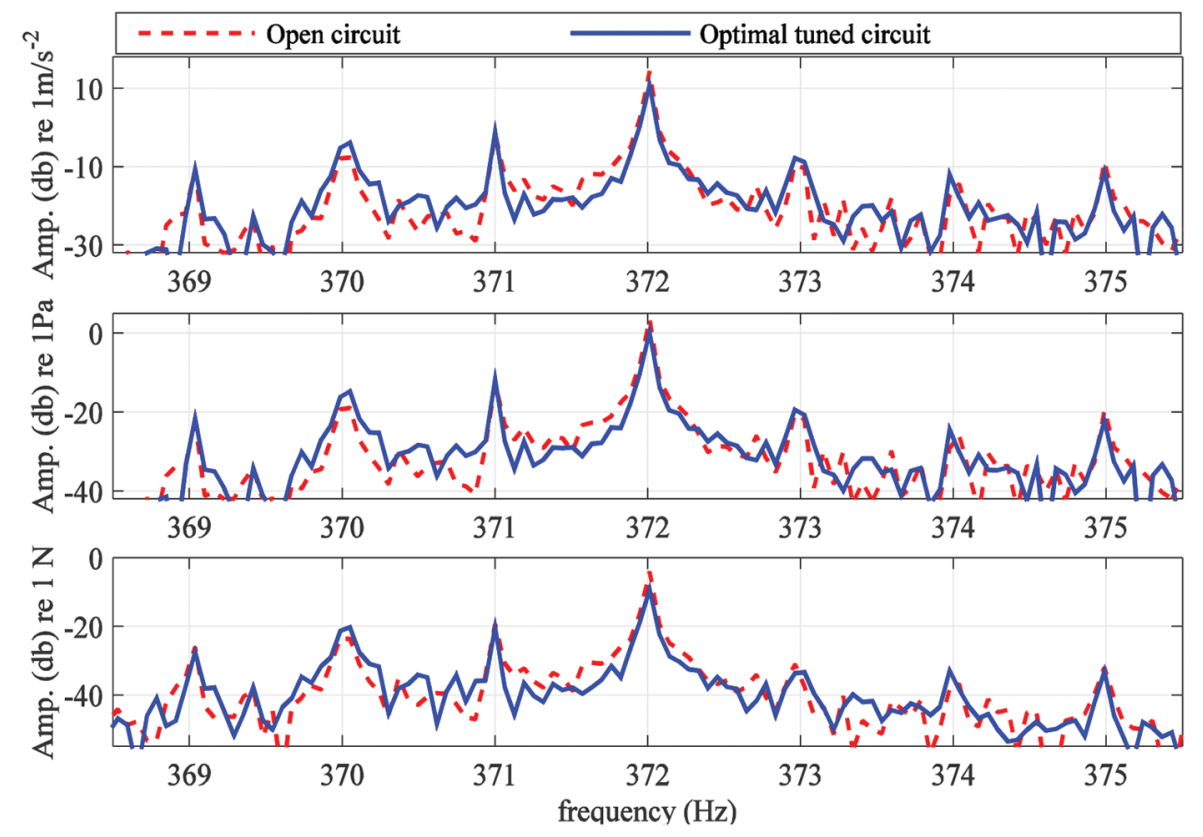

Figure I I. Comparison of, from top to bottom, the plate vibration, transmitted force and noise radiation with mistuned and optimally tuned shunt circuit in the frequency domain.
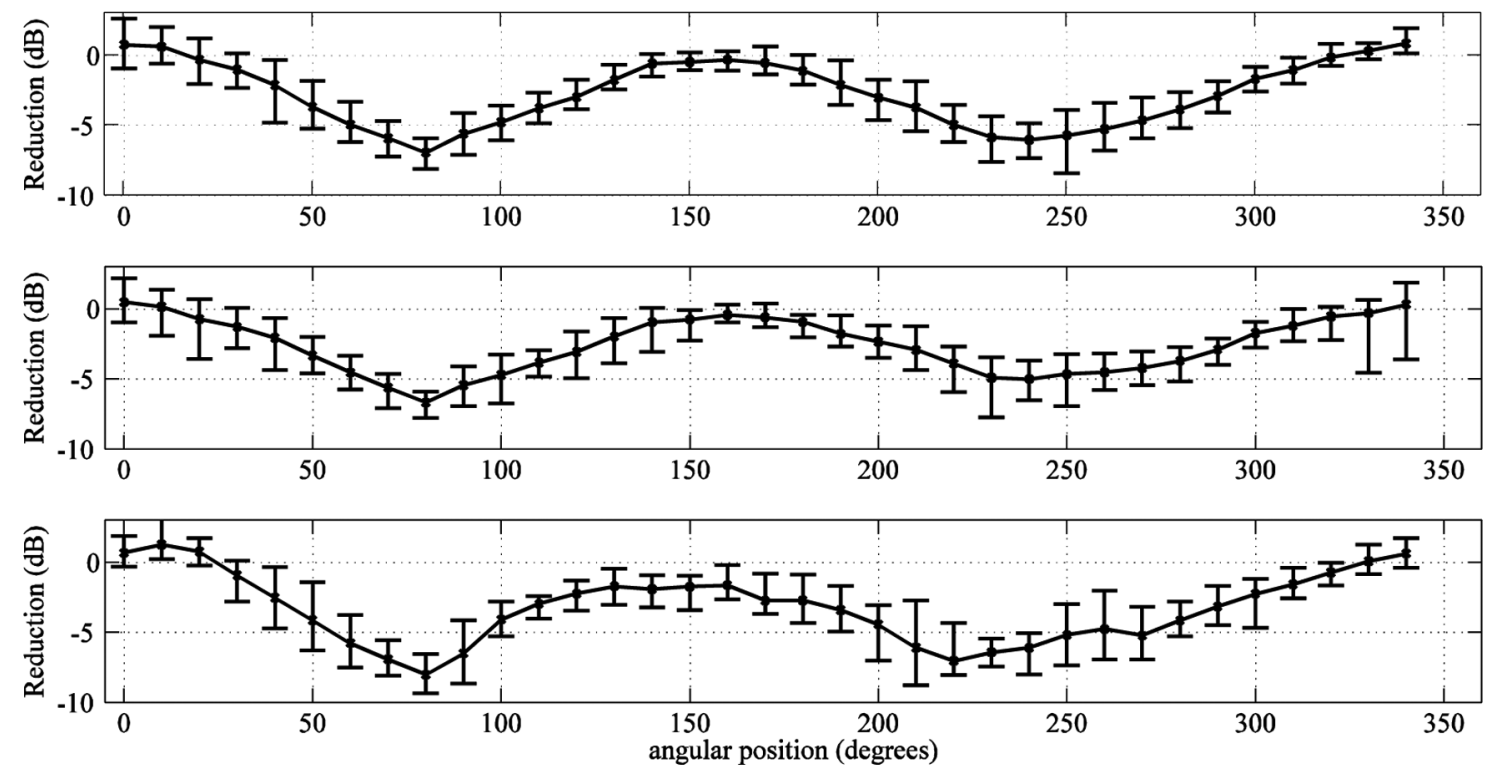

Figure 12. Reductions of, from top to bottom, the plate vibration, transmitted force and noise radiation in the angular domain.

inductance shunt circuit mistuned, then optimally tuned at $t=23 \mathrm{~s}$ and then mistuned again at $t=46 \mathrm{~s}$. The time domain signals of the first two segments (mistuned and optimally tuned) are then projected into the frequency domain and compared in Figure 11. Here, a reduction of $3.5 \mathrm{~dB}$ is obtained for all three signals at $372 \mathrm{~Hz}$, but an undesired amplification of around $3 \mathrm{~dB}$ shows up at $370 \mathrm{~Hz}$ which is two rotating speed harmonics below the excitation frequency. In order to further interpret the obtained results, the achieved reductions are also plotted as a function of the angular position of the shaft. To do so, the time domain signals are synchronised with the rotating shaft speed signal measured by the encoder, and the reductions are calculated in an interval of $10^{\circ}$, as shown in Figure 12. In these figures, the average reductions are represented by the dottedline and the variations during different revolutions are indicated as the error bars. As can be seen, the maximum reduction is obtained at the angular positions of $80^{\circ}$ and $240^{\circ}$, around which the piezoelectric actuator is almost parallel to the disturbance force. With the shaft position around $0 / 360^{\circ}$ and $180^{\circ}$, where the PBRIA line 

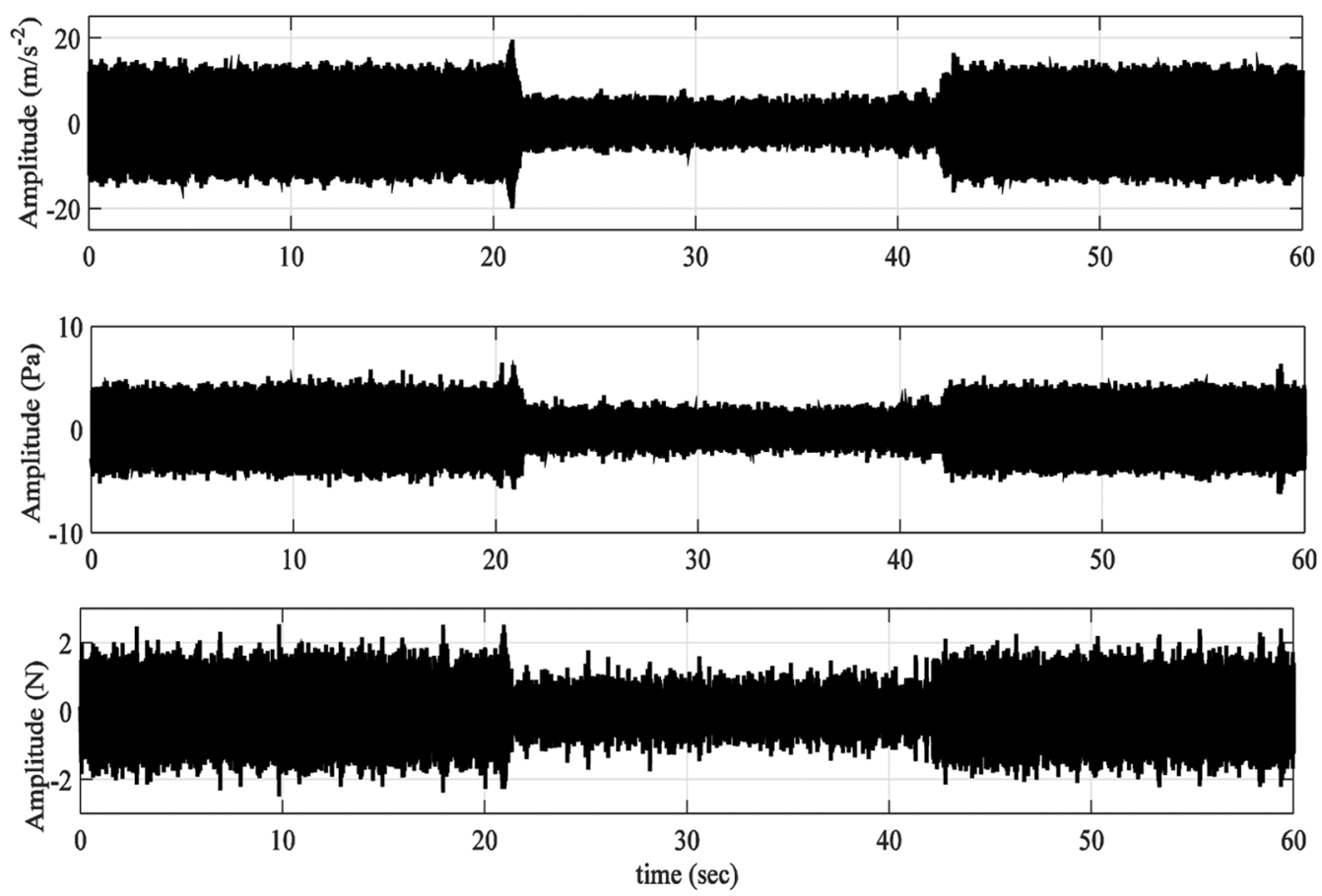

Figure 13. From top to bottom, the plate acceleration, the transmitted force and the radiated noise in the time domain, with and without optimal inductance tuning.
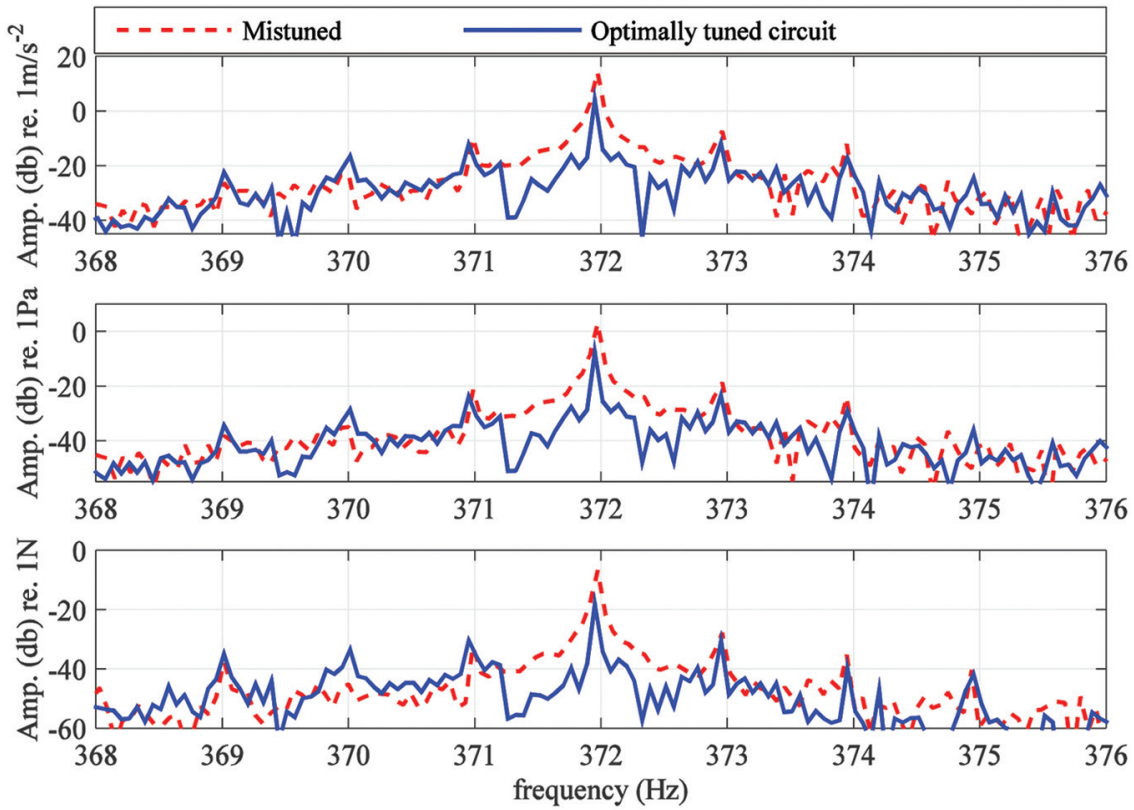

Figure 14. Comparison of, from top to bottom, the plate vibration, transmitted force and noise radiation with mistuned and optimally tuned shunt circuit in the frequency domain.

of action is perpendicular to the disturbance, there are nearly no reductions.

In the second set of the experiments, both resonantly shunted PBRIAs are activated, while the setup still rotates at $60 \mathrm{r} / \mathrm{min}$. The measured plate vibration, transmitted force and the radiated noise are plotted in Figure 13, where the shunt circuits are first mistuned, then optimally tuned and finally mistuned again. Here, the reduction for each measured signal is clearly noticeable, especially compared to the case where only one PBRIA was activated. The corresponding amplitude spectra of the three measured responses in the first two segments are again compared in Figure 14. A reduction of about $11 \mathrm{~dB}$ of the transmitted force is obtained at 

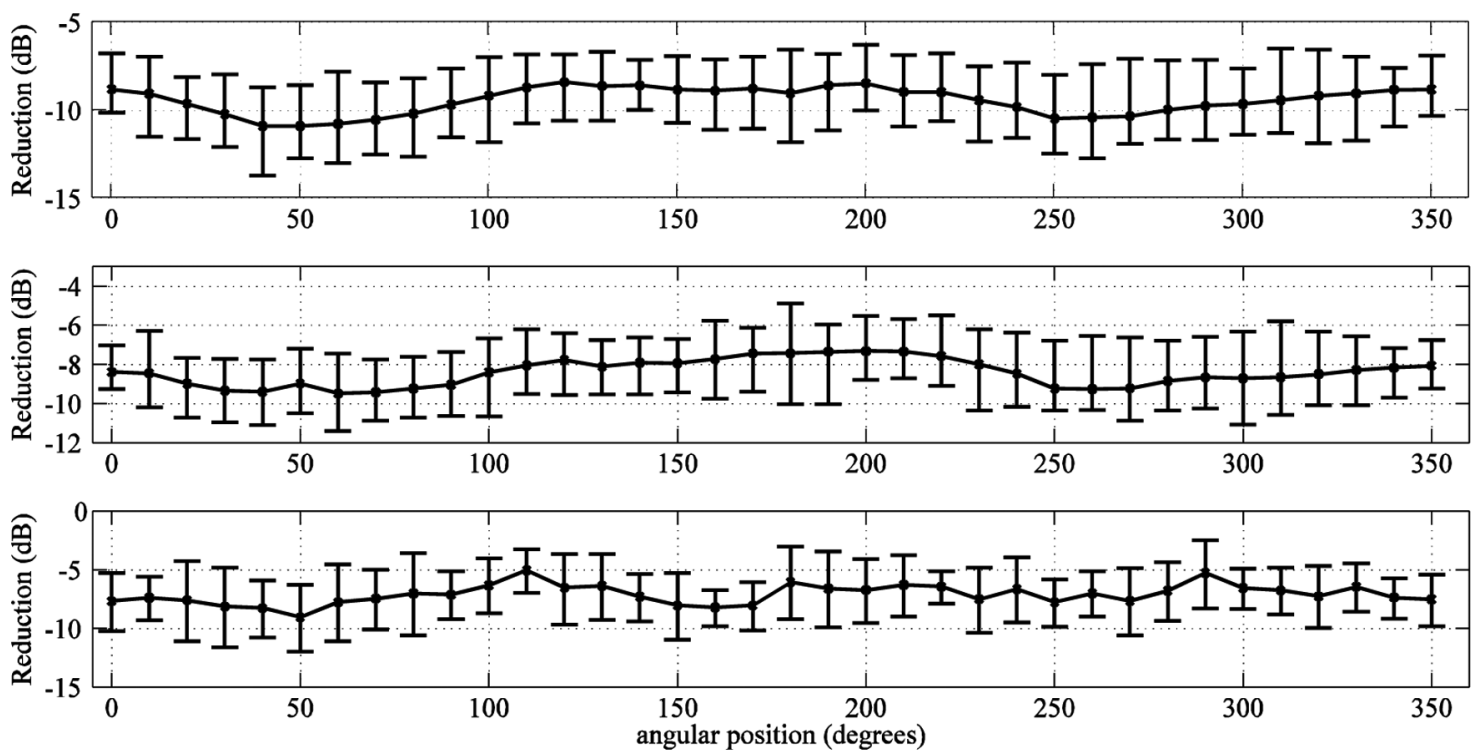

Figure I5. Reductions of, from top to bottom, the plate vibration, transmitted force and noise radiation in the angular domain.

the excitation frequency, which leads to a reduction of $9 \mathrm{~dB}$ for the plate vibration and the radiated noise. The control effect is then again examined in the angular domain in Figure 15. It is clear that the control approach is effective at any angular position, although it exhibits a small modulation of the averaged achievable reductions. A comparison of the achieved reduction curves depicted in Figures 12 and 15 with the curves in Figure 3 indicates that the modelling method is able to qualitatively predict performances of the resonantly shunted PBRIAs.

\section{Conclusion}

This article has discussed a novel approach for suppressing rotating machinery radiating noise by using shunted PBRIAs that can rotate together with the machinery. It has been shown that in principle the radiated noise can be controlled by suppressing the disturbance force transmitted to the housing. A bidirectional lumped parameter model of a simplified system is presented, which consists of a rotating shaft and two perpendicularly mounted resonantly shunted PBRIAs, in order to investigate the theoretically achievable performance. The proposed approach has also been validated on an experimental test bed. When the shaft is not rotating, a significant reduction of noise and vibrations is obtained at the resonant frequencies of the system, using only a single PBRIA. When the shaft rotates, good reductions are obtained, but now two perpendicularly mounted PBRIAs are needed. These results demonstrate the technical feasibility of using the considered PBRIAs for suppressing structureborne noise of rotating machinery. The obtained results also correspond well with predictions from the simplified bi-directional lumped parameter model.

\section{Acknowledgements}

The China Scholarship Council is gratefully acknowledged.

\section{Declaration of conflicting interests}

The author(s) declared no potential conflicts of interest with respect to the research, authorship and/or publication of this article.

\section{Funding}

The author(s) disclosed receipt of the following financial support for the research, authorship, and/or publication of this article: The research was supported by the IWT Flanders within the OptiWind project (GA: IWT/120029), the Research Fund KU Leuven and the European Commission within the ITN EMVeM Marie Curie project (GA: 315967). The European Commission is gratefully acknowledged for their support of the ANTARES research project (GA 606817). The EU H2020 Grant No. 606817, the IWT Flanders within the ASTRA project, the ITEA2 through the MODRIO project are also gratefully acknowledged for its support.

\section{References}

Alujević N, Gardonio P and Frampton KD (2011) Smart double panel for the sound radiation control: blended velocity feedback. AIAA Journal 49(6): 1123-1134.

Alujević N, Tomac I and Gardonio P (2012) Tuneable vibration absorber using acceleration and displacement feedback. Journal of Sound and Vibration 331(12): 2713-2728. 
Alujević N, Zhao G, Depraetere B, et al. (2014) $\mathcal{H}_{2}$ optimal vibration control using inertial actuators and a comparison with tuned mass dampers. Journal of Sound and Vibration 333(18): 4073-4083.

Chen MH and Brennan MJ (2000) Active control of gear vibration using specially configured sensors and actuators. Smart Materials and Structures 9(3): 342-350.

Crawley E and De Luis J (1987) Use of piezoelectric actuators as elements of intelligent structures. AIAA Journal 25: 1373-1385.

Date M, Kutani M and Sakai S (2000) Electrically controlled elasticity utilizing piezoelectric coupling. Journal of Applied Physics 87(2): 863-868.

Davis CL and Lesieutre GA (2000) An actively tuned solidstate vibration absorber using capacitive shunting of piezoelectric stiffness. Journal of Sound and Vibration 232(3): 601-617.

Dehandschutter W (1997) The Reduction of Structure-Borne Noise by Active Control of Vibration. Leuven: KU Leuven.

Den Hartog JP (1985) Mechanical Vibrations (Civil, Mechanical and Other Engineering Series). New York: Dover Publications.

Devos S and Pinte G (2009) Extension of Final Report on Active Damping. Leuven: Flanders' Mechatronics Technology Centre (FMTC).

Elliott SJ, Boucher CC and Nelson PA (1992) The behavior of a multiple channel active control system. IEEE Transactions on Signal Processing 40(5): 1041-1052.

Elliott SJ, Nelson PA, Stothers IM, et al. (1990) In-flight experiments on the active control of propeller-induced cabin noise. Journal of Sound and Vibration 140(2): 219-238.

Fuller CR, Elliott SJ and Nelson PA (1997) Active Control of Vibration. London: Academic Press.

Guan YH, Li M, Lim TC, et al. (2004) Comparative analysis of actuator concepts for active gear pair vibration control. Journal of Sound and Vibration 269(1-2): 273-294.

Guan YH, Lim TC and Shepard WS (2005) Experimental study on active vibration control of a gearbox system. Journal of Sound and Vibration 282(3-5): 713-733.

Hagood NW and Von Flotow A (1991) Damping of structural vibrations with piezoelectric materials and passive electrical networks. Journal of Sound and Vibration 146(2): 243-268.

Hollkamp JJ (1994) Multimodal passive vibration suppression with piezoelectric materials and resonant shunts. Journal of Intelligent Material Systems and Structures 5(1): 49-57.

Hollkamp JJ and Starchville TF (1994) A self-tuning piezoelectric vibration absorber. Journal of Intelligent Material Systems and Structures 5(4): 559-566.

Hunt JB (1979) Dynamic Vibration Absorbers. London: Mechanical Engineering Publications.

Inman DJ (2008) Engineering Vibration. Upper Saddle River, NJ: Pearson Prentice Hall.

Kodejska M, Mokry P, Linhart V, et al. (2012) Adaptive vibration suppression system: an iterative control law for a piezoelectric actuator shunted by a negative capacitor. IEEE Transactions on Ultrasonics, Ferroelectrics and Frequency Control 59(12): 2785-2796.

Kuo SM and Morgan D (1995) Active Noise Control Systems: Algorithms and DSP Implementations. 1st ed. New York: John Wiley.
Lesieutre GA (1994) Vibration damping and control using shunted piezoelectric materials. The Shock and Vibration and Digest 30(3): 187-195.

Li M, Lim TC, Shepard WS, et al. (2005) Experimental active vibration control of gear mesh harmonics in a power recirculation gearbox system using a piezoelectric stack actuator. Smart Materials and Structures 14(5): 917-927.

Nelson PA and Elliott SJ (1991) Active Control of Sound. New York: Academic Press.

Neubauer M, Oleskiewicz R, Popp K, et al. (2006) Optimization of damping and absorbing performance of shunted piezo elements utilizing negative capacitance. Journal of Sound and Vibration 298(1-2): 84-107.

Niederberger D (2005) Smart Damping Materials using Shunt Control. Göttingen: Cuvillier Verlag.

Park CH and Inman DJ (2003) Enhanced piezoelectric shunt design. Shock and Vibration 10(2): 127-133.

Paulitsch C, Gardonio P and Elliott SJ (2006) Active vibration control using an inertial actuator with internal damping. The Journal of the Acoustical Society of America 119(4): 2131-2140.

Paulitsch C, Gardonio P, Elliott SJ, et al. (2004) Design of a lightweight, electrodynamic, inertial actuator with integrated velocity sensor for active vibration control of a thin lightly-damped panel. In: International conference on noise and vibration engineering. Leuven: KU Leuven. Available at: https://www.isma-isaac.be/publications/PMA_MOD_publications/ISMA2004/239_254.pdf

Pinte G (2007) Active Control of Repetitive Impact Noise. Leuven: KU Leuven.

Pinte G, Devos S, Stallaert B, et al. (2010) A piezo-based bearing for the active structural acoustic control of rotating machinery. Journal of Sound and Vibration 329(9): 1235-1253.

Preumont A (2002) Vibration Control of Active Structures: An Introduction. Dordrecht: Kluwer Academic Publishers.

Qiu Z, Zhang X, Wu H, et al. (2007) Optimal placement and active vibration control for piezoelectric smart flexible cantilever plate. Journal of Sound and Vibration 301(3-5): 521-543.

Rebbechi B, Howard C and Hansen C (1999) Active control of gearbox vibration. In: Proceedings of the active control of sound and vibration conference, 2-4 December, Fort Lauderdale, FL, pp. 295-304. Available at: http://data.mecheng.adelaide.edu.au/avc/publications/public_papers/ 1999/active99cqh.pdf

Stallaert B (2010) Active Structural Acoustic Source Control of Rotating Machinery. Leuven: KU Leuven.

Sutton TJ, Elliott SJ, Brennan MJ, et al. (1997) Active isolation of multiple structural waves on a helicopter gearbox support strut. Journal of Sound and Vibration 205(1): $81-101$.

Zhao G (2015) Active structural acoustic source control of rotating machinery using piezo-based rotating inertial actuators. PhD Thesis, KU Leuven.

Zhao G, Alujević N, Depraetere B, et al. (2014) Dynamic analysis and $\mathcal{H}_{2}$ optimisation of a piezo-based tuned vibration absorber. Journal of Intelligent Material Systems and Structures. Epub ahead of print 14 August. DOI: 10.1177/ 1045389 X14546652. 


\section{Appendix I}

The mass matrix $\{M\}_{\theta}$ defined in equation (3) is diagonal and it is populated as follows

$M_{6 \times 6}=$
$\left[\begin{array}{cccccc}m_{1} & m_{2} & m_{3} & 0 & 0 & 0 \\ 0 & m_{2} \cos (\theta) & 0 & 0 & m_{2} \sin (\theta) & 0 \\ 0 & 0 & 0 & 0 & 0 & 0 \\ 0 & 0 & 0 & m_{1} & m_{2} & m_{3} \\ 0 & 0 & m_{3} \sin (\theta) & 0 & 0 & -m_{3} \cos (\theta) \\ 0 & 0 & 0 & 0 & 0 & 0\end{array}\right]$

The matrix $\{C\}_{\theta}$ is given by

$$
C_{6 \times 6}=\left[\begin{array}{ccccc}
c_{1,1} & c_{1,2} & c_{1,3} & \cdots & c_{1,6} \\
c_{2,1} & c_{2,2} & c_{2,3} & \cdots & c_{2,6} \\
c_{3,1} & c_{3,2} & c_{3,3} & \cdots & c_{3,6} \\
\cdots & \cdots & \cdots & \cdots & \cdots \\
c_{6,1} & c_{6,2} & c_{6,3} & \cdots & c_{6,6}
\end{array}\right]
$$

The elements of equation (11) are

$$
c_{1,1-6}=\left[\begin{array}{llllll}
c_{4} & 0 & 0 & 0 & 0 & 0
\end{array}\right]
$$

$c_{2,1 \sim 6}=c_{2}\left[\begin{array}{llllll}-\cos (\theta) & \cos (\theta) & 0 & -\sin (\theta) & \sin (\theta) & 0\end{array}\right]$

$$
\begin{aligned}
& c_{3,1 \sim 6}=\left[\begin{array}{llllll}
0 & 0 & 0 & 0 & 0 & 0
\end{array}\right] \\
& c_{4,1-6}=\left[\begin{array}{llllll}
0 & 0 & 0 & c_{1} & 0 & 0
\end{array}\right]
\end{aligned}
$$

$$
c_{6,1 \sim 6}=\left[\begin{array}{llllll}
0 & 0 & 0 & 0 & 0 & 0
\end{array}\right]
$$

The matrix $\{K\}_{\theta}$ is given by

$$
K_{6 \times 6}=\left[\begin{array}{ccccc}
k_{1,1} & k_{1,2} & k_{1,3} & \cdots & k_{1,6} \\
k_{2,1} & k_{2,2} & k_{2,3} & \cdots & k_{2,6} \\
k_{3,1} & k_{3,2} & k_{3,3} & \cdots & k_{3,6} \\
\cdots & \cdots & \cdots & \cdots & \cdots \\
k_{6,1} & k_{6,2} & k_{6,3} & \cdots & k_{6,6}
\end{array}\right]
$$

The elements of equation (18) are

$$
k_{1,1-6}=\left[\begin{array}{llllll}
k_{4} & 0 & 0 & 0 & 0 & 0
\end{array}\right]
$$

$$
\begin{aligned}
& k_{2,1 \sim 6}=\left(\begin{array}{lllll}
k_{2}+k_{p 1}^{*}
\end{array}\right) \\
& {\left[\begin{array}{llllll}
-\cos (\theta) & \cos (\theta) & 0 & -\sin (\theta) & \sin (\theta) & 0
\end{array}\right]}
\end{aligned}
$$

$k_{3,1 \sim 6}=\left[\begin{array}{llllll}\sin (\theta) & -\sin (\theta) & 0 & -\cos (\theta) & \cos (\theta) & 0\end{array}\right]$

$$
\begin{aligned}
& k_{4,1 \sim 6}=\left[\begin{array}{llllll}
0 & 0 & 0 & k_{1} & 0 & 0
\end{array}\right] \\
& k_{5,1-6}=\left(k_{3}+k_{p 2}^{*}\right) \\
& {\left[\begin{array}{llllll}
\sin (\theta) & 0 & -\sin (\theta) & -\cos (\theta) & 0 & \cos (\theta)
\end{array}\right]}
\end{aligned}
$$

$k_{6,1 \sim 6}=\left[\begin{array}{llllll}\cos (\theta) & 0 & -\cos (\theta) & \sin (\theta) & 0 & -\sin (\theta)\end{array}\right]$

The displacement vector is populated as follows

$$
u_{6 \times 1}=\left[\begin{array}{llllll}
x_{1} & x_{2} & x_{3} & y_{1} & y_{2} & y_{3}
\end{array}\right]^{T}
$$

The force vector takes the form

$$
F_{d}(s)_{6 \times 1}=\left[\begin{array}{llllll}
0 & 0 & 0 & 1 & 0 & 0
\end{array}\right]^{T}
$$

